\title{
Integrated Management of Turmeric Rhizome Rot Caused by Pythium aphanidermatum
}

\author{
P.G. Chavan*, K.T. Apet and R.S. Borade \\ Department of Plant Pathology, Vasantrao Naik Marathwada Krishi Vidyapeeth, \\ Parbhani - 431 402, Maharashtra, India \\ *Corresponding author
}

\section{A B S T R A C T}

\begin{tabular}{|l|}
\hline Key w or d s \\
$\begin{array}{l}\text { Pythium } \\
\text { aphanidermatum, } \\
\text { Turmeric. }\end{array}$ \\
\hline Article Info \\
\hline $\begin{array}{l}\text { Accepted: } \\
\text { 30 September } 2017 \\
\text { Available Online: } \\
\text { 10 November } 2017\end{array}$ \\
\hline \hline
\end{tabular}

Turmeric (Curcuma longa L) rhizome rot caused by Pythium aphanidermatum was one of the divesting disease and causes accountable losses. All fungicides, bioagents, botanicals and soil amendments (alone and in combination) tested in vitro for management of turmeric rhizome rot was found effective against $P$. aphanidermatum. However, significantly highest reduction in average mortality was recorded with Metalaxyl (RT) + its drenching $(85.37 \%)$, followed by T4 + T5 (RT) + T1 (SA) (74.54 \%), Carbendazim 12 $\% \mathrm{WP}+$ Mancozeb $63 \% \mathrm{WP}(\mathrm{RT})+$ its drenching $(73.30 \%), \mathrm{T} 4$ + T5 (RT) + T3 (SA) $(71.87 \%)$, Bioagent consortia (RT) + Its Drenching $71.20 \%)$, Copper oxychloride (RT) + its drenching $(68.79 \%), \mathrm{T} 4+\mathrm{T} 5(\mathrm{RT})+\mathrm{T} 2(\mathrm{SA})(68.40 \%), \mathrm{T} 4+\mathrm{T} 5(\mathrm{RT})+$ Bioagent consortia (SA) (68.07 \%), Metalaxyl (RT) (68.80\%), Carbendazim $12 \%$ WP + Mancozeb $63 \%$ WP (RT) (62.60\%), Copper oxychloride (RT) (61.31\%), Trichoderma viride (RT) $(54.86 \%)$, Neem leaf extract (RT) (51.22\%) and NSKE (SA) (48.09\%).

\section{Introduction}

Turmeric e belongs to family Zingiberaceae. This is also called as 'hidden Lilly' or 'golden spice' or 'turmeric of commerce' or 'Indian saffron' or 'Haldi'. It is originated from Tropical South Asia. Turmeric is the third largest spice produced in the country and it accounts for about $14 \%$ of total spices produced in India. India is the world's largest producer of turmeric and apparently accounts for more than 80 per cent of the world's production, followed by China, Indonesia, Bangladesh, and Thailand (Selvan et al., 2002). The area, production and productivity of turmeric in India has been reported to be 175.73 and 185.58 thousand hectares, 959.35 and 943.33 thousand tones and 5459 and 5083 $\mathrm{kg} / \mathrm{ha}$, respectively, during year 2014-15 and 2015-16 (Anonymous, 2016). The total area in Maharashtra under turmeric was 11.0 thousand hectares, with production 11.0 thousand tones and productivity of 1000 $\mathrm{kg} / \mathrm{ha}$, respectively (Anonymous, 2015).

Turmeric is prone to many fungal, bacterial, viral and nematode diseases. Among all diseases rhizome rot caused by $P$. aphanidermatum is most destructive and widespread disease causes very high crop loss under favorable conditions (Rathaiah, 1982). The disease has been reported to causes more than 60 per cent mortality of seedlings both in nursery and field condition and about 50-80 
per cent losses during storage (Nirmal, 1992); rhizome rot resulted in yield loss of $50 \%$ (Rajalakshmi et al., 2016).

\section{Materials and Methods}

On the basis of results obtained in in vitro (plate and pot culture) the fungicides, bioagents, botanicals and soil amendments found effective were selected for integrated management of turmeric rhizome rot (pot culture). The earthen pots $(30 \mathrm{~cm}$ dia.) disinfected with 5 per cent of Copper sulphate solution were filled with the autoclaved potting mixture of soil: sand: FYM (2:1:1). The mass multiplied (sand: maize medium) inoculum of $P$. aphanidermatum was inoculated (@50 g / kg potting mixture) separately to the potting mixture in pots, mixed thoroughly, watered adequately and incubated for two weeks in the screen house, to proliferate the pathogen and make the soil / potting mixture sick. The pot culture experiment comprised of 15 treatments as described under treatment details.

The test fungicides, talc based formulations of the bioagents and consortia, aqueous extract of botanicals and soil amendments were applied (alone and in combination) as presowing rhizome treatment to the healthy rhizomes of turmeric Cv. Selum and post sowing soil application. After 96 hrs treated healthy rhizomes of turmeric $\mathrm{Cv}$. Selum were sown (1 rhizome / pot), in the earthen pots containing $P$. aphanidermatum sick soil and maintained in the screen house. The earthen pots containing $P$. aphanidermatum sick soil sown with surface sterilized healthy rhizomes of turmeric Cv. Selum and without application of any treatment were maintained as untreated control. For each treatment, six pots / treatment / replication were maintained and all treatments replicated thrice. All these pots were watered regularly and maintained in the screen house for further studies.
Observations on rhizome germination and pre emergence rhizome rot (PERR) will be recorded at 30 days after sowing and that of post emergence seedling mortality (PESM) at $60,90,120$ and 150 DAS. The per cent of rhizome germination, pre emergence rhizome rot (PERR) and post emergence seedling mortality (PESM) will be calculated by following formulae.

No. of rhizomes germinated

Germination $(\%)=$

Total no. of rhizomes sown

No. of rhizomes ungerminated

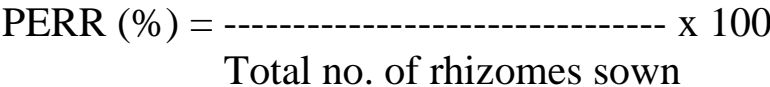

No. of seedlings died

$\operatorname{PESM}(\%)=\underset{\text { Total no. of seedlings }}{\text {----- }}$

\section{Results and Discussion}

\section{Effect on rhizome germination}

Results (Table 1 and Fig. 1) revealed that all the test treatments improved rhizome germination, over untreated control and it was ranged from 65.14 [NSKE (SA)] to 90.17 (Metalaxyl (RT) + its drenching) per cent, as against 40.06 per cent in untreated control.

However it was significantly highest with Metalaxyl (RT) + its drenching (90.17 \%), followed by Metalaxyl (RT) (83.71\%), T4 + T5 (RT) + T1 (SA) (80.41\%), Carbendazim $12 \% \mathrm{WP}+$ Mancozeb $63 \% \mathrm{WP}(\mathrm{RT})+$ its drenching (80.12\%), T4 + T5 (RT) + T3 (SA) (79.86\%), Bioagent consortia (RT) + its drenching (79.52\%), Copper oxychloride (RT) + its drenching (77.94\%), T4 + T5 (RT) + T2 (SA) (77.68\%), T4 + T5 (RT) + Bioagent consortia (SA) (77.54 \%), Carbendazim $12 \%$ WP + Mancozeb $63 \%$ WP (RT) (77.48 \%), Copper oxychloride 
(RT) $(76.14 \%)$ and Trichoderma viride (RT) (70.92\%). Whereas, NSKE (SA) and Neem leaf extract (RT) were found least effective with comparatively minimum rhizome germination of 65.14 and 67.56 per cent, respectively. Further, all the test treatments recorded significantly increase in rhizome germination, over untreated control and it was ranged from 38.50 [NSKE (SA)] to 55.57 (Metalaxyl (RT) + its drenching) per cent. However, highest increase in rhizome germination was recorded with Metalaxyl (RT) + its drenching $(55.57 \%)$, followed by Metalaxyl (RT) (52.14\%), T4 + T5 (RT) + T1 (SA) $(50.18 \%)$, Carbendazim $12 \% \mathrm{WP}+$ Mancozeb $63 \%$ WP (RT) + its drenching $(50.00 \%), \mathrm{T} 4+\mathrm{T} 5(\mathrm{RT})+\mathrm{T} 3(\mathrm{SA})(49.84$ $\%)$, Bioagent consortia (RT) + its drenching (49.62\%), Copper oxychloride (RT) + its drenching (48.60\%), T4 + T5 (RT) + T2 (SA) $(48.43 \%), \mathrm{T} 4+\mathrm{T} 5(\mathrm{RT})+$ Bioagent consortia (SA) $(48.34 \%)$, Carbendazim $12 \% \mathrm{WP}+$ Mancozeb 63 \% WP (RT) (48.30\%), Copper oxychloride (RT) $(47.39 \%)$ and Trichoderma viride (RT) (43.51\%). Whereas, NSKE (SA) and Neem leaf extract (RT) were found less effective with 38.50 and 40.70 per cent increase in rhizome germination, respectively.

\section{Effect on pre and post emergence mortalities}

It is evident from Table 1 and Figure 1 all the treatments significantly influenced the both pre emergence rhizome rot (PERR) and post emergence seedling mortality (PESM), caused by $P$. aphanidermatum in turmeric Cv. Selum.

The pre emergence rhizome rot (PERR) recorded with all the treatments was ranged from 9.83 (Metalaxyl (RT) + its drenching) to 34.86 [NSKE (SA)] per cent, as against 59.94 per cent in untreated control. However it was significantly least with Metalaxyl (RT) + its drenching $(9.83 \%)$, followed by Metalaxyl (RT) $(16.29 \%), \mathrm{T} 4+\mathrm{T} 5(\mathrm{RT})+\mathrm{T} 1(\mathrm{SA})$
(19.59 \%), Carbendazim $12 \%$ WP + Mancozeb $63 \%$ WP (RT) + its drenching $(19.88 \%), \mathrm{T} 4$ + T5 (RT) + T3 (SA) $(20.14$ $\%)$, Bioagent consortia (RT) + its drenching (20.48\%), Copper oxychloride (RT) + its drenching $(22.06 \%), \mathrm{T} 4+\mathrm{T} 5(\mathrm{RT})+\mathrm{T} 2(\mathrm{SA})$ $(22.32 \%), \mathrm{T} 4+\mathrm{T} 5(\mathrm{RT})+$ Bioagent consortia (SA) $(22.46 \%)$, Carbendazim $12 \% \mathrm{WP}+$ Mancozeb $63 \%$ WP (RT) (22.52\%), Copper oxychloride (RT) (23.86\%) and Trichoderma viride (RT) (29.08\%). Whereas, NSKE (SA) and Neem leaf extract (RT) were found least effective with comparatively maximum pre emergence rhizome rot of 34.86 and 32.44 per cent, respectively.

The post emergence seedling mortality (PESM) recorded with all the treatments was ranged from 12.85 (Metalaxyl (RT) + its drenching) to 35.23 [NSKE (SA)] per cent, as against 100 per cent in untreated control. However it was significantly least with Metalaxyl (RT) + its drenching (12.85\%), followed by T4 + T5 (RT) + T1 (SA) (18.23 $\%$ ), Carbendazim $12 \%$ WP + Mancozeb 63 $\% \mathrm{WP}(\mathrm{RT})+$ its drenching (20.24 \%), T4 + T5 (RT) + T3 (SA) (22.65 \%), Bioagent consortia (RT) + its drenching (23.43\%), Copper oxychloride (RT) + its drenching $(25.61 \%), \mathrm{T} 4+\mathrm{T} 5(\mathrm{RT})+\mathrm{T} 2(\mathrm{SA})(25.97$ $\%), \mathrm{T} 4+\mathrm{T} 5(\mathrm{RT})+$ Bioagent consortia (SA) (26.38 \%), Metalaxyl (RT) (35.23 \%), Carbendazim $12 \%$ WP + Mancozeb $63 \%$ WP (RT) (37.22 \%), Copper oxychloride (RT) $(37.58 \%)$ and Trichoderma viride (RT) $(41.76 \%)$. Whereas, NSKE (SA) and Neem leaf extract (RT) were found least effective with comparatively maximum post emergence seedling mortality of 45.67 and 43.44 per cent, respectively.

The average mortality recorded with all the treatments was ranged from 11.34 (Metalaxyl (RT) + its drenching) to 40.27 [NSKE (SA)] per cent, as against 79.97 per cent in untreated control. 
Table.1 Integrated disease management against turmeric rhizome rot Cv. Selum (Pot culture)

\begin{tabular}{|c|c|c|c|c|c|c|c|c|c|c|}
\hline \multirow{2}{*}{$\begin{array}{l}\text { Tr. } \\
\text { No. }\end{array}$} & \multirow{2}{*}{ Treatments } & \multirow{2}{*}{$\begin{array}{c}\text { Dose (g/kg of } \\
\text { rhizome or t/ha of } \\
\text { soil) }\end{array}$} & \multirow{2}{*}{$\begin{array}{c}\text { Germination * } \\
(\%)\end{array}$} & \multirow[t]{2}{*}{$\begin{array}{c}\text { \% Incr. } \\
\text { over contr. }\end{array}$} & \multicolumn{2}{|c|}{$\begin{array}{c}\text { Incidence } \\
(\%) *\end{array}$} & \multirow{2}{*}{$\begin{array}{c}\text { Av. Mor. } \\
\qquad(\%)\end{array}$} & \multicolumn{2}{|c|}{ Red. $(\%)$ over control } & \multirow{2}{*}{$\begin{array}{c}\text { Av. Red. } \\
(\%)\end{array}$} \\
\hline & & & & & PERR & PESM & & PERR & PESM & \\
\hline $\mathrm{T}_{1}$ & Metalaxyl (RT) & $1.0 \mathrm{~g} / \mathrm{kg}$ & $\begin{array}{c}83.71 \\
(66.20)\end{array}$ & $\begin{array}{c}52.14 \\
(46.23) \\
\end{array}$ & $\begin{array}{c}16.29 \\
(23.80)\end{array}$ & $\begin{array}{c}35.23 \\
(36.41)\end{array}$ & $\begin{array}{c}25.76 \\
(30.50) \\
\end{array}$ & $\begin{array}{c}72.82 \\
(58.58)\end{array}$ & $\begin{array}{c}64.77 \\
(53.59) \\
\end{array}$ & $\begin{array}{c}68.80 \\
(56.04)\end{array}$ \\
\hline $\mathrm{T}_{2}$ & Copper oxychloride (RT) & $2.5 \mathrm{~g} / \mathrm{kg}$ & $\begin{array}{c}76.14 \\
(60.76) \\
\end{array}$ & $\begin{array}{c}47.39 \\
(43.50) \\
\end{array}$ & $\begin{array}{c}23.86 \\
(29.24) \\
\end{array}$ & $\begin{array}{c}37.58 \\
(37.81) \\
\end{array}$ & $\begin{array}{c}30.72 \\
(33.66) \\
\end{array}$ & $\begin{array}{c}60.19 \\
(50.88) \\
\end{array}$ & $\begin{array}{c}62.42 \\
(52.19) \\
\end{array}$ & $\begin{array}{c}61.31 \\
(51.53) \\
\end{array}$ \\
\hline $\mathrm{T}_{3}$ & $\begin{array}{l}\text { Carbendazim } 12 \% \text { WP + Mancozeb } \\
63 \% \text { WP (RT) }\end{array}$ & $2.0 \mathrm{~g} / \mathrm{kg}$ & $\begin{array}{r}77.48 \\
(61.67) \\
\end{array}$ & $\begin{array}{c}48.30 \\
(44.02) \\
\end{array}$ & $\begin{array}{c}22.52 \\
(28.33) \\
\end{array}$ & $\begin{array}{c}37.22 \\
(37.60) \\
\end{array}$ & $\begin{array}{c}29.87 \\
(33.13) \\
\end{array}$ & $\begin{array}{c}62.43 \\
(52.20) \\
\end{array}$ & $\begin{array}{c}62.78 \\
(52.40) \\
\end{array}$ & $\begin{array}{c}62.60 \\
(52.30) \\
\end{array}$ \\
\hline $\mathrm{T}_{4}$ & Trichoderma viride (RT) & $10 \mathrm{~g} / \mathrm{kg}$ & $\begin{array}{c}70.92 \\
(57.37) \\
\end{array}$ & $\begin{array}{c}43.51 \\
(41.27) \\
\end{array}$ & $\begin{array}{l}29.08 \\
(32.63) \\
\end{array}$ & $\begin{array}{c}41.76 \\
(40.26) \\
\end{array}$ & $\begin{array}{c}35.42 \\
(36.52) \\
\end{array}$ & $\begin{array}{c}51.48 \\
(45.85) \\
\end{array}$ & $\begin{array}{c}58.24 \\
(49.74) \\
\end{array}$ & $\begin{array}{c}54.86 \\
(47.79) \\
\end{array}$ \\
\hline $\mathrm{T}_{5}$ & Neem leaf extract (RT) & $10 \mathrm{ml} / \mathrm{kg}$ & $\begin{array}{c}67.56 \\
(55.28) \\
\end{array}$ & $\begin{array}{c}40.70 \\
(39.64) \\
\end{array}$ & $\begin{array}{c}32.44 \\
(34.72) \\
\end{array}$ & $\begin{array}{c}43.44 \\
(41.23) \\
\end{array}$ & $\begin{array}{c}37.94 \\
(38.02) \\
\end{array}$ & $\begin{aligned} 45.88 \\
(42.64) \\
\end{aligned}$ & $\begin{array}{c}56.56 \\
(48.77) \\
\end{array}$ & $\begin{array}{c}51.22 \\
(45.70) \\
\end{array}$ \\
\hline $\mathrm{T}_{6}$ & NSKE (SA) & $\begin{array}{l}50 \mathrm{~g} / \mathrm{Kg} \text { of potting } \\
\text { mixture }\end{array}$ & $\begin{array}{c}65.14 \\
(53.81) \\
\end{array}$ & $\begin{array}{c}38.50 \\
(38.35) \\
\end{array}$ & $\begin{array}{c}34.86 \\
(36.19) \\
\end{array}$ & $\begin{array}{c}45.67 \\
(42.52) \\
\end{array}$ & $\begin{array}{c}40.27 \\
(39.39) \\
\end{array}$ & $\begin{array}{c}41.84 \\
(40.30) \\
\end{array}$ & $\begin{array}{c}54.33 \\
(47.48) \\
\end{array}$ & $\begin{array}{c}48.09 \\
(43.90) \\
\end{array}$ \\
\hline $\mathrm{T}_{7}$ & Metalaxyl $(\mathrm{RT})+$ its drenching & $1.0 \mathrm{~g} / \mathrm{kg}+1.0 \%$ & $\begin{array}{c}90.17 \\
(71.73) \\
\end{array}$ & $\begin{array}{c}55.57 \\
(48.20) \\
\end{array}$ & $\begin{array}{c}9.83 \\
(18.28) \\
\end{array}$ & $\begin{array}{l}12.85 \\
(21.01) \\
\end{array}$ & $\begin{array}{c}11.34 \\
(19.68) \\
\end{array}$ & $\begin{array}{r}83.59 \\
(66.11) \\
\end{array}$ & $\begin{array}{c}87.15 \\
(68.99) \\
\end{array}$ & $\begin{array}{r}85.37 \\
(67.51) \\
\end{array}$ \\
\hline $\mathrm{T}_{8}$ & $\begin{array}{l}\text { Copper oxychloride }(\mathrm{RT})+\text { its } \\
\text { drenching }\end{array}$ & $2.5 \mathrm{~g} / \mathrm{kg}+2.5 \%$ & $\begin{array}{r}77.94 \\
(61.99) \\
\end{array}$ & $\begin{array}{c}48.60 \\
(44.20) \\
\end{array}$ & $\begin{array}{l}22.06 \\
(28.01) \\
\end{array}$ & $\begin{array}{c}25.61 \\
(30.40) \\
\end{array}$ & $\begin{array}{c}23.84 \\
(29.22) \\
\end{array}$ & $\begin{array}{r}63.20 \\
(52.65) \\
\end{array}$ & $\begin{array}{r}74.39 \\
(59.60) \\
\end{array}$ & $\begin{array}{c}68.79 \\
(56.04) \\
\end{array}$ \\
\hline $\mathrm{T}_{9}$ & $\begin{array}{l}\text { Carbendazim } 12 \% \mathrm{WP}+\text { Mancozeb } \\
63 \% \mathrm{WP}(\mathrm{RT})+\text { its drenching } \\
\end{array}$ & $2.0 \mathrm{~g} / \mathrm{kg}+2.0 \%$ & $\begin{array}{c}80.12 \\
(63.52) \\
\end{array}$ & $\begin{array}{c}50.00 \\
(45.00) \\
\end{array}$ & $\begin{array}{c}19.88 \\
(26.48) \\
\end{array}$ & $\begin{array}{c}20.24 \\
(26.74) \\
\end{array}$ & $\begin{array}{l}20.06 \\
(26.61) \\
\end{array}$ & $\begin{array}{c}66.83 \\
(54.84) \\
\end{array}$ & $\begin{array}{c}79.76 \\
(63.26) \\
\end{array}$ & $\begin{array}{r}73.30 \\
(58.89) \\
\end{array}$ \\
\hline $\mathrm{T}_{10}$ & $\mathrm{~T} 4+\mathrm{T} 5(\mathrm{RT})+\mathrm{T} 1(\mathrm{SA})$ & $\begin{array}{c}10 \mathrm{~g} / \mathrm{kg}+10 \mathrm{ml} / \mathrm{kg} \\
+1.0 \%\end{array}$ & $\begin{array}{c}80.41 \\
(63.73)\end{array}$ & $\begin{array}{c}50.18 \\
(45.10) \\
\end{array}$ & $\begin{array}{l}19.59 \\
(26.27)\end{array}$ & $\begin{array}{c}18.23 \\
(25.28)\end{array}$ & $\begin{array}{c}18.91 \\
(25.78)\end{array}$ & $\begin{array}{c}67.32 \\
(55.13) \\
\end{array}$ & $\begin{array}{c}81.77 \\
(64.72)\end{array}$ & $\begin{array}{c}74.54 \\
(59.70)\end{array}$ \\
\hline $\mathrm{T}_{11}$ & $\mathrm{~T} 4+\mathrm{T} 5(\mathrm{RT})+\mathrm{T} 2(\mathrm{SA})$ & $\begin{array}{c}10 \mathrm{~g} / \mathrm{kg}+10 \mathrm{ml} / \mathrm{kg} \\
+2.5 \%\end{array}$ & $\begin{array}{c}77.68 \\
(61.81) \\
\end{array}$ & $\begin{array}{c}48.43 \\
(44.10) \\
\end{array}$ & $\begin{array}{c}22.32 \\
(28.19) \\
\end{array}$ & $\begin{array}{r}25.97 \\
(30.64) \\
\end{array}$ & $\begin{array}{l}24.15 \\
(29.43) \\
\end{array}$ & $\begin{array}{r}62.76 \\
(52.39) \\
\end{array}$ & $\begin{array}{r}74.03 \\
(59.36) \\
\end{array}$ & $\begin{array}{r}68.40 \\
(55.79) \\
\end{array}$ \\
\hline $\mathrm{T}_{12}$ & $\mathrm{~T} 4+\mathrm{T} 5(\mathrm{RT})+\mathrm{T} 3(\mathrm{SA})$ & $\begin{array}{c}10 \mathrm{~g} / \mathrm{kg}+10 \mathrm{ml} / \mathrm{kg} \\
+2.0 \%\end{array}$ & $\begin{aligned} 79.86 \\
(63.33) \\
\end{aligned}$ & $\begin{array}{c}49.84 \\
(44.91) \\
\end{array}$ & $\begin{array}{l}20.14 \\
(26.67) \\
\end{array}$ & $\begin{array}{c}22.65 \\
(28.42) \\
\end{array}$ & $\begin{array}{l}21.40 \\
(27.55) \\
\end{array}$ & $\begin{array}{r}66.40 \\
(54.57) \\
\end{array}$ & $\begin{array}{r}77.35 \\
(61.58) \\
\end{array}$ & $\begin{array}{l}71.87 \\
(57.97) \\
\end{array}$ \\
\hline $\mathrm{T}_{13}$ & $\begin{array}{l}\mathrm{T} 4+\mathrm{T} 5(\mathrm{RT})+\text { Bioagent consortia } \\
(\mathrm{SA})\end{array}$ & $\begin{array}{c}10 \mathrm{~g} / \mathrm{kg}+10 \mathrm{ml} / \mathrm{kg} \\
+4 \mathrm{~kg} / \mathrm{ha}\end{array}$ & $\begin{array}{r}77.54 \\
(61.71) \\
\end{array}$ & $\begin{array}{c}48.34 \\
(44.05) \\
\end{array}$ & $\begin{array}{l}22.46 \\
(28.29) \\
\end{array}$ & $\begin{array}{c}26.38 \\
(30.90) \\
\end{array}$ & $\begin{array}{c}24.42 \\
(29.61) \\
\end{array}$ & $\begin{array}{c}62.53 \\
(52.26) \\
\end{array}$ & $\begin{array}{r}73.62 \\
(59.10) \\
\end{array}$ & $\begin{array}{c}68.07 \\
(55.60) \\
\end{array}$ \\
\hline $\mathrm{T}_{14}$ & $\begin{array}{l}\text { Bioagent consortia }(\mathrm{RT})+\text { its } \\
\text { drenching }\end{array}$ & $10 \mathrm{~g} / \mathrm{kg}+4 \mathrm{~kg} / \mathrm{ha}$ & $\begin{array}{r}79.52 \\
(63.09) \\
\end{array}$ & $\begin{array}{c}49.62 \\
(44.78) \\
\end{array}$ & $\begin{array}{c}20.48 \\
(26.91) \\
\end{array}$ & $\begin{array}{r}23.43 \\
(28.95) \\
\end{array}$ & $\begin{array}{l}21.96 \\
(27.94) \\
\end{array}$ & $\begin{array}{r}65.83 \\
(54.23) \\
\end{array}$ & $\begin{array}{c}76.57 \\
(61.05) \\
\end{array}$ & $\begin{array}{r}71.20 \\
(57.54) \\
\end{array}$ \\
\hline $\mathrm{T}_{15}$ & Control & & $\begin{array}{c}40.06 \\
(39.27) \\
\end{array}$ & $\begin{array}{c}00.00 \\
(00.00) \\
\end{array}$ & $\begin{array}{c}59.94 \\
(50.73) \\
\end{array}$ & $\begin{array}{l}100.00 \\
(90.00) \\
\end{array}$ & $\begin{array}{c}79.97 \\
(63.41) \\
\end{array}$ & $\begin{array}{c}00.00 \\
(00.00) \\
\end{array}$ & $\begin{array}{c}00.00 \\
(00.00) \\
\end{array}$ & $\begin{array}{c}00.00 \\
(00.00) \\
\end{array}$ \\
\hline & $\mathbf{S E} \pm$ & & 0.26 & 0.24 & 0.31 & 0.30 & 0.31 & 0.38 & 0.30 & 0.34 \\
\hline & $\mathrm{CD}(\mathrm{P}=0.01)$ & & 0.74 & 0.70 & 0.90 & 0.86 & 0.88 & 1.02 & 0.86 & 0.94 \\
\hline
\end{tabular}

*-Mean of three replications, Av.: Average, Mor.: Concentration, Incr.: Increase Red.: Reduction, PERR: Pre emergence rhizome rot, PESM: Post Emergence Seedling Mortality, RT: Rhizome Treatment, SA: Soil Application, Figures in parentheses are angular transformed values 
Fig.1 Integrated efficacy of various treatments against turmeric rhizome rot (Pot Culture)

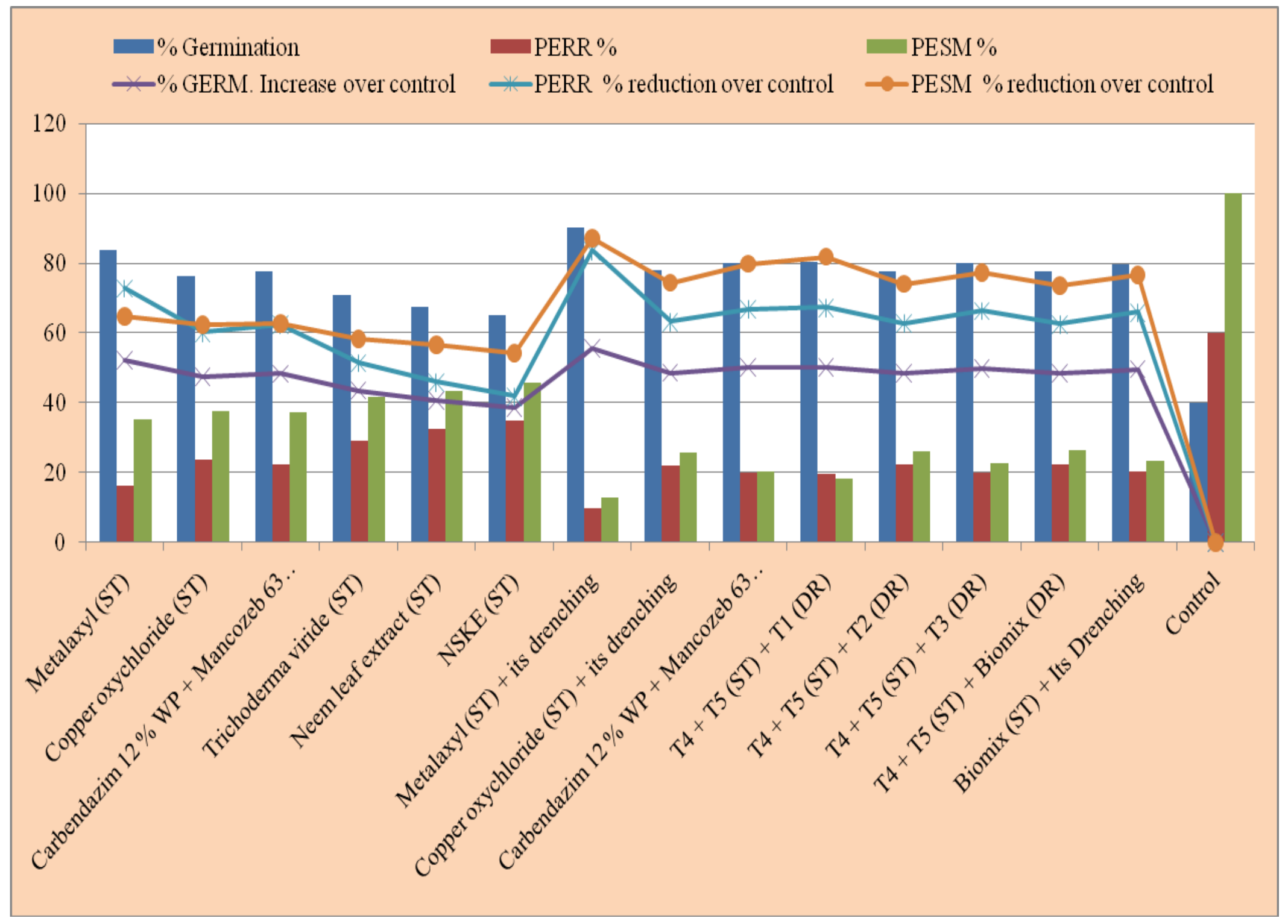


However, it was significantly least with Metalaxyl (RT) + its drenching (11.34 \%), followed by T4 + T5 (RT) + T1 (SA) (18.91 $\%)$, Carbendazim $12 \% \mathrm{WP}+$ Mancozeb 63 $\% \mathrm{WP}(\mathrm{RT})+$ its drenching $(20.06 \%)$, T4 + T5 (RT) + T3 (SA) (21.40\%), Bioagent consortia (RT) + its drenching (21.96\%), Copper oxychloride (RT) + its drenching $(23.84 \%), \mathrm{T} 4+\mathrm{T} 5(\mathrm{RT})+\mathrm{T} 2(\mathrm{SA})(24.15$ $\%), \mathrm{T} 4+\mathrm{T} 5(\mathrm{RT})+$ Bioagent consortia (SA) (24.42 \%), Metalaxyl (RT) (25.76 \%), Carbendazim $12 \%$ WP + Mancozeb $63 \%$ WP (RT) (29.87 \%), Copper oxychloride (RT) $(30.72 \%)$ and Trichoderma viride (RT) $(35.42 \%)$. Whereas, NSKE (SA) and Neem leaf extract (RT) were found least effective with comparatively maximum average mortality of 40.27 and 37.94 per cent, respectively.

\section{Reduction in mortality}

All the test treatments were found to reduce both PERR and PESM over untreated control (Table 1).

The reduction in pre emergence rhizome rot (PERR) was ranged from 41.84 [NSKE (SA)] to 83.59 (Metalaxyl (RT) + its drenching) per cent. However, it was significantly highest with Metalaxyl (RT) + its drenching (83.59 $\%$ ), followed by Metalaxyl (RT) (72.82 \%), $\mathrm{T} 4$ + T5 (RT) + T1 (SA) (67.32\%), Carbendazim $12 \%$ WP + Mancozeb $63 \%$ WP (RT) + its drenching (66.83\%), T4 + T5 $(\mathrm{RT})+\mathrm{T} 3$ (SA) $(66.40 \%)$, Bioagent consortia (RT) + its drenching (65.83\%), Copper oxychloride $(\mathrm{RT})+$ its drenching $(63.20 \%)$, $\mathrm{T} 4+\mathrm{T} 5(\mathrm{RT})+\mathrm{T} 2(\mathrm{SA})(62.76 \%), \mathrm{T} 4+\mathrm{T} 5$ (RT) + Bioagent consortia (SA) (62.53\%), Carbendazim $12 \%$ WP + Mancozeb $63 \%$ WP (RT) (62.43 \%), Copper oxychloride (RT) $(60.19 \%)$ and Trichoderma viride (RT) $(51.48 \%)$. Whereas, NSKE (SA) and Neem leaf extract (RT) were recorded comparatively least reduction in pre emergence rhizome rot of 41.84 and 45.88 per cent, respectively.

The reduction in post emergence seedling mortality (PESM) was ranged from 54.33 [NSKE (SA)] to 87.15 (Metalaxyl (RT) + its drenching) per cent. However, it was significantly highest with Metalaxyl (RT) + its drenching $(87.15 \%)$, followed by T4 + T5 $(\mathrm{RT})+\mathrm{T} 1$ (SA) (81.77\%), Carbendazim 12 $\% \mathrm{WP}+$ Mancozeb $63 \% \mathrm{WP}(\mathrm{RT})+$ its drenching (79.76\%), T4 + T5 (RT) + T3 (SA) (77.35\%), Bioagent consortia (RT) + its drenching (76.57\%), Copper oxychloride (RT) + its drenching (74.39\%), T4 + T5 (RT) + T2 (SA) (74.03\%), T4 + T5 (RT) + Bioagent consortia (SA) (73.62\%), Metalaxyl (RT) (64.77\%), Carbendazim $12 \% \mathrm{WP}+$ Mancozeb $63 \%$ WP (RT) (62.78\%), Copper oxychloride (RT) (62.42\%) and Trichoderma viride (RT) (58.24\%). Whereas, NSKE (SA) and Neem leaf extract (RT) were recorded comparatively least reduction in post emergence seedling mortality of 54.33 and 56.56 per cent, respectively.

The reduction in average mortality was ranged from 48.09 [NSKE (SA)] to 85.37 (Metalaxyl (RT) + its drenching) per cent. However, it was significantly highest with Metalaxyl (RT) + its drenching (85.37\%), followed by T4 + T5 (RT) + T1 (SA) $(74.54$ $\%$ ), Carbendazim $12 \% \mathrm{WP}+$ Mancozeb 63 \% WP (RT) + its drenching (73.30\%), T4 + T5 (RT) + T3 (SA) (71.87 \%), Bioagent consortia (RT) + its drenching $71.20 \%$ ), Copper oxychloride (RT) + its drenching $(68.79 \%), \mathrm{T} 4+\mathrm{T} 5(\mathrm{RT})+\mathrm{T} 2(\mathrm{SA})(68.40$ $\%), \mathrm{T} 4+\mathrm{T} 5(\mathrm{RT})+$ Bioagent consortia (SA) (68.07 \%), Metalaxyl (RT) (68.80 \%), Carbendazim $12 \%$ WP + Mancozeb $63 \%$ WP (RT) (62.60 \%), Copper oxychloride (RT) (61.31\%) and Trichoderma viride (RT) (54.86 \%). Whereas, NSKE (SA) and Neem leaf extract (RT) were recorded comparatively least reduction in average mortality of 48.09 and 51.22 per cent, respectively. These results are in conformity with the findings of those reported earlier by several workers against, Pythium aphanidermatum infecting turmeric (Sagar, 2006; Bharathi and Sudhakar, 2011; 
Shanmugam et al., 2015), P. aphanidermatum infecting ginger (Jayasekhar et al., 2000; Balakrishnan, 2005; Anonymous, 2006; Sagar, 2006; Kulkarni et al., 2007; Kulkarni, 2011; Kadam, 2014, Basistha and Homan, 2015; Dhroo et al., 2015).

\section{References}

Anonymous, 2006. Annual Report 2004-2005, All India Coordinated Research Project on Spices, Kasaragod, Kerala, India, pp-18.

Anonymous, 2015. Horticultural Statistics at a Glance. Pp-225.

Anonymous, 2016. Spices Area, Production and Productivity in India, All India Coordinated Research Project on Spices, Kasaragod, Kerala, India, pp-1.

Balakrishnan, P., 2005. Bio ecology of rhizome rot pathogen(s) of ginger and disease management, Technical Bulletin No. 8, Abstracts of M.Sc. and Ph.D Dissertations on spice crops, pp-71.

Basistha, A., and Homan, R. 2015. Evaluation and selection of appropriate management package of ginger rhizome rot disease in field condition. J. Agric. Vet. Sci., 8 (6): 53-56.

Bharathi, V., and Sudhakar, R. 2011. Management of rhizome rot of turmeric (Curcuma longa $\mathrm{L}$.) through IDM. Ann. Pl. Protec. Sci., 19 (2): 400-402.

Dohroo, N. P., Kansal, S. and Ahluwalia N. 2015. Studies on eco farmer friendly practices for management of soft rot of ginger (Zingiber officinale). Ind. Phytopath, 68 (1): 93-96.

Jayasekhar, M., Joshua, J. P. and Pillai, O. A. 2000. Management of rhizome rot of ginger caused by Pythium aphanidermatum. Madras Agri. J., 87(1/3): 170-171.

Kadam, R. V., 2014. Investigations on rhizome rot of ginger. M.Sc. (Agri.) thesis submitted to Vansantro Naik Marathwada Krishi
Vidyapeeth, Parbhani.

Kulkarni, S., 2011. Etiology, epidemiology and integrated management of rhizome rot of ginger in Karnataka. Ind. Phytopath. 64 (2): $113-119$

Kulkarni, S., Sagar, D., Kulkarni, S. A., Hegde, G. M., Hegde, R. V. and Lokesha. N. M. 2007. Investigations on the etiology and integrated management of rhizome rot of ginger and turmeric in Karnataka, Technical Bulletin-4, Dept. of Plant Pathology, UAS, Dharwad, Karnataka, India., pp-34.

Nirmal, B. K., Samsuddin, K. and Ratnambal, M. J. 1992. In vitro plant regeneration from leaf derived callus in ginger (Zingiber officinale Rosc.). Plant, Cell Tissue and Organ Culture, 29: 71-74.

Rajalakshmi, J., Durgadevi, D., Harish, S. and Raguchander, T. 2016. Morphological and molecular characterization of Pythium aphanidermatum the incitant of rhizome rot in turmeric. Int. J. Env. Ecol. Family and Urban Studies, 6 (4): 1-8.

Rathaiah, Y., 1982. Rhizome rot of turmeric. Ind. Phytopath, 35: 415-417.

Sagar, S. D., 2006. Investigations on the etiology, epidemiology and integrated management of rhizome rot complex of ginger and turmeric. Ph.D. Thesis submitted to University of Agriculture, Dharwad.

Selvan, M. T., Thomas, K. G. and Manojkumar, K. 2002. Ginger (Zingiber officinale Rosc.) In: Indian Spices-Production and utilization (Singh, H. P., Sivaraman K and Selvan M. T., Eds.) Coconut development Board, Calicut, 110-131.

Shanmugam, P. S., Indhumathi, K., Sangeetha, M. and Tamilselvan, N. 2015. Evaluation of different pest management modules against major insect pests and diseases of turmeric. Current Biotica, 9 (1): 17-24.

\section{How to cite this article:}

Chavan, P.G., K.T. Apet and Borade, R.S. 2017. Integrated Management of Turmeric Rhizome Rot Caused by Pythium aphanidermatum. Int.J.Curr.Microbiol.App.Sci. 6(11): 5321-5327. doi: https://doi.org/10.20546/ijcmas.2017.611.508 